\title{
Análise de objetivos e conclusões de estudos com nove plantas usadas para o controle de diabetes em Mato Grosso
}

\author{
Analysis of objectives and study findings with nine plants used for diabetes \\ control in Mato Grosso
}

DOI $10.5935 / 2446-4775.20160027$

\author{
'SANTOS, Ana Paula A. dos*; ${ }^{2}$ RIEDER, Arno \\ ${ }^{1}$ Universidade do Estado de Mato Grosso, UNEMAT; Rondônia, Brasil. \\ ${ }^{2}$ Universidade do Estado de Mato Grosso, UNEMAT; Mato Grosso, Brasil \\ *Correspondências: anap andrade.cba@hotmail.com
}

\section{Resumo}

Diabetes é uma doença metabólica que ocorre devido à destruição das células pancreáticas ou falha na ação/secreção de insulina, causando hiperglicemia e outras complicações. $O$ uso terapêutico de plantas representa prática antiga, em geral, de baixo custo e acessível. Este trabalho analisa os objetivos e conclusões de estudos sobre nove plantas medicinais usadas na terapia do diabetes, no estado de Mato Grosso. Os estudos foram recuperados na internet, por meio das ferramentas do Google Acadêmico, utilizando-se quatro modalidades de buscas e o nome científico das plantas, com cinco variantes da palavra diabetes. Dessas buscas foram aproveitados 208 artigos, que foram lançados em planilha de Excel e analisados em programa estatístico apropriado. As abordagens predominantes foram as que citam a aplicação da planta para diabetes $(31,8 \%)$ e efeito glicemiante (16,4\%). Para 111 estudos com bioatividade detectada, 108 foram favoráveis e 3 não favoráveis à terapia do diabetes. Nas espécies Sambucus australis Cham. \& Schltdl e Vitex cymosa Bertero ex Spreng houve constatação de uso popular para a diabetes; para Sambucus nigra L. verificou-se a potencialização da insulina; para Alternanthera brasiliana (L.) Kuntze, houve efeito na cicatrização de feridas e, para as espécies: Cecropia pachystachya Trécul, Eryngium foetidum L., Scoparia dulcis L. e Stevia rebaudiana (Bertoni) Bertoni houve constatação de efeito hipoglicemiante.

Palavras-chave: Glicemiante. Fitoquímica. Mato Grosso. Controle do diabetes. Plantas medicinais. Scoparia dulcis. Stevia rebaudiana.

\begin{abstract}
Diabetes is a metabolic disease which occurs due to destruction of pancreatic cells, or failure in the action/insulin secretion, causing hyperglycemia and other complications. The therapeutic use of medicinal plants represents an old practice and generally is inexpensive and accessible. The study reveals the
\end{abstract}


objectives and conclusions of studies on nine plants used in Mato Grosso applied in diabetes therapy. Data were retrieved on the Internet, through Google Scholar, using four search modes, scientific name of plants with five variants of the word diabetes. From these searches, there were intended for 208 articles, released in Excel and their analysis on appropriate statistical program. The prevalent approach was mentioning application of the plant for diabetes (31.8\%) and glicemiante effect (16.4\%). To 111 studies with bioactivity detected, 108 were favorable and 3 non-favorable to diabetes therapy. In Sambucus australis Cham. \& Schltdl and Vitex cymosa Bertero ex Spreng species were popular use of observation for diabetes; Sambucus nigra L. found insulin potentiation; with Alternanthera brasiliana (L.) Kuntze, there was no effect on wound healing and for species: Cecropia pachystachya Trécul, Eryngium foetidum L., Scoparia dulcis L. and Stevia rebaudiana (Bertoni) Bertoni was hypoglycemic effect of observation.

Keywords: Glicemiante. Phytochemistry. Mato Grosso. Diabetes control. Medicinal plants. Scoparia dulcis. Stevia rebaudiana.

\section{Introdução}

Conforme Rieder e Guarim Neto (2012), diabetes Mellitus (DM) é uma síndrome ou doença crônica, autoimune, associada a distúrbios metabólicos dos carboidratos, lipídios e proteínas, instalando-se também desequilíbrios eletrolíticos.

De acordo com as diretrizes da Sociedade Brasileira de Diabetes, o DM é um grupo heterogêneo de distúrbios metabólicos tendo em comum a hiperglicemia, resultante de problemas insulínicos (defeitos na ação e/ou secreção de insulina). Descrevem que os principais tipos de DM são: DM tipo 1), onde há destruição das células beta suprimindo a produção de insulina; este tipo pode ser subdivido em subtipos $1 \mathrm{~A}$ e $1 \mathrm{~B}$; - o DM $1 \mathrm{~A}$ é autoimune abrangendo 5 a $10 \%$ dos casos; nesta há a destruição de células betapancreáticas imunomediada; - o DM 1B é a idiopática sendo menos frequente; nesta há ausência de marcadores de autoimunidade contra as células beta e, não havendo associação a haplótipos do sistema HLA. DM tipo 2) neste tipo ocorre em $90 \%-95 \%$ dos casos, causado por defeito insulínico; DM gestacional que se desenvolve intolerância à glicose, de amplitude variável, sendo diagnosticada durante a gestação (SBD, 2016). Ainda segundo a mesma, o número de pessoas diabéticas aumenta em decorrência de fatores como o envelhecimento e crescimento populacional, a maior urbanização, a crescente prevalência de obesidade e sedentarismo, assim como aumento da sobrevida de diabéticos.

Segundo a Organização Mundial de Saúde (OMS, 2002), o uso da medicina tradicional é generalizado nos países em desenvolvimento, enquanto o uso de medicina complementar e alternativa está aumentando rapidamente nos países desenvolvidos. Em muitas partes do mundo, os responsáveis políticos, profissionais de saúde e o público em geral debatem-se com questões acerca da segurança, eficácia, qualidade, disponibilidade, preservação e desenvolvimento deste tipo de recursos e atendimento.

A medicina tradicional inclui terapias que fazem uso da medicação onde são usados remédios com base em ervas, partes de animais e/ou minerais e terapias sem medicação que são realizadas principalmente sem o uso de fármaco, como é o caso da acupuntura (OMS, 2002). Já a medicina complementar e alternativa é definida pelo The National Center for Complementary and Alternative Medicine (NCCAM), nos 
Estados Unidos da América, como um grupo de diversos sistemas médicos e de saúde, práticas e produtos que não são atualmente considerados como parte de medicina convencional (CAM, 2008).

O que move as pessoas a adotar práticas, integrativas e complementares em saúde, é o desejo de participar ativamente de processos que revelam outras formas de aprender, praticar e cuidar da saúde própria e dos outros (TELESI JUNIOR, 2016). Diante disso, os produtos com origem de plantas medicinais ocupam um espaço cada vez maior no mercado (CORRÊA e ALVES, 2008).

Mazzolin (2013), pesquisando potencial antioxidante em plantas, menciona que os desafios da ciência incluem a compreensão dos mecanismos envolvidos para intervir, com terapêuticas alternativas novas e mais eficazes.

Devido à escassez de estudos clínicos com plantas medicinais é importante levar em conta que algumas podem ter efeitos tóxicos, como por exemplo, hepatotoxidez e bloqueio $\beta$ adrenérgico, que podem levar o efeito desejado a hipoglicemia, não como método terapêutico eficaz, mas tóxico. Desse modo, há necessidade de encontrar plantas medicinais que ofereçam eficácia terapêutica e saúde. A toxidez também pode ser influenciada pela parte da planta utilizada na preparação do extrato, método e via de administração (NEGRI, 2005).

Os avanços em pesquisa sobre fitoterápicos em focos farmacológicos, toxicológicos e moleculares permitiram constatar mecanismo de ação total ou parcialmente esclarecido, com avaliação toxicológica segura, ou não (YUNES, PEDROSA e CECHINEL FILHO, 2001). De acordo com os autores, grande parte dos fitoterápicos fabricados pela indústria brasileira está fundamentada apenas no uso popular da planta medicinal, sem comprovação pré-clínica ou clínica, não sendo competitivos em âmbito nacional ou internacional, o que pode levá-los a desaparecer da indústria nacional de fitoterápicos. Chaves (2012), estudando plantas para terapia do lúpus, cita que no Brasil a grande maioria da população não tem acesso a medicamentos, que, além de apresentarem custo elevado, também, apresentam efeitos indesejáveis adversos. Os resultados do uso de plantas e seus metabólitos, alcançados até o momento, estimulam a pesquisa de novos medicamentos fitoterápicos para terapias.

O presente artigo tem por finalidade analisar objetivos, visando o estudo de nove plantas medicinais utilizadas para o controle do diabetes e suas respectivas conclusões.

\section{Materiais e Métodos}

Os propósitos deste estudo se limitaram as seguintes famílias de espécies de plantas medicinais aplicadas para o controle do diabetes no estado de Mato Grosso, da ampla lista indicada em Rieder e Guarim Neto (2012): Amaranthaceae: Alternanthera brasiliana (L.) Kuntze; Urticaceae: Cecropia pachystachya Trécul; Alismataceae: Echinodorus macrophyllus (Kunth) Micheli; Apiaceae: Eryngium foetidum L.; Adoxaceae: Sambucus australis Cham. \& Schltdl; Adoxaceae: Sambucus nigra L.; Plantaginaceae: Scoparia dulcis L.; Asteraceae: Stevia rebaudiana (Bertoni) Bertoni; Lamiaceae: Vitex cymosa Bertero ex Spreng. Este trabalho analisa objetivos e conclusões constantes em publicações de estudos com estas plantas para diabetes ou abordagens associadas a esta. As publicações foram resgatadas via internet contida em base de dados, buscadas pelas ferramentas e recursos do Google Acadêmico. 
Em conformidade com a metodologia definida por Rieder e Rodrigues (2012), foram utilizadas quatro modalidades de buscas por publicações sobre diabetes, em bases de acesso parcial ou totalmente livre, disponíveis na internet. Na busca utilizou-se a palavra-chave "diabetes" e em seguida também as suas variantes: "diabetic", "antidiabetic", "hypoglycemic", "hyperglycemic" e "antihyperglycemic". As modalidades de busca foram: M3 (notítulo: "nome científico" "variantes"); M4 (notítulo: "nome científico" "variantes" "abstract"); M5 (tudonotítulo: "nome científico" "variantes") e M6 ("nome científico" "variantes" "abstract").

As buscas foram realizadas no período de 03 jul. 2013 à 03 fev. 2014. Os textos recuperados foram lidos, e os condizentes com os propósitos, tiveram os dados lançados em um banco eletrônico apropriado (Excel), para alimentar as análises. O banco resultante foi composto por 208 publicações.

Os critérios para inclusão foram de estudos publicados em "sites" de abordagem científica, encontrados pelas ferramentas do Google Acadêmico, que apresentaram vínculo direto ou indireto entre o diabetes e as espécies envolvidas. Entende-se por vínculo direto, artigos que abordaram diretamente em seu estudo a planta para o diabetes e; indireto, artigos que não visaram diretamente a planta para o diabetes, mas que se referiam a outros estudos, estes sim investigando a planta para o diabetes. Textos aproveitados são aqueles que se referem, em seus estudos, a essas nove plantas medicinais usadas na atividade antidiabética ou, para alguma complicação associada ou, ainda, usos para tais fins.

As análises referem-se aos objetivos dos estudos e respectivas conclusões constantes nos textos publicados e aproveitados. Foram utilizadas ferramentas do Excel e de um programa estatístico apropriado, aplicando recursos analíticos de síntese e de interpretação.

\section{Resultados e Discussão}

Os resultados que tratam dos objetivos e conclusões de 208 publicações reunidas nesse trabalho estão apresentados nas TABELAS 1, 2 E 3.

TABELA 1. Objetivo dos estudos indicados nos textos aproveitados que se referem à fitoterapia do diabetes, com nove espécies utilizadas em Mato Grosso (MT), Brasil (BR), [Buscas na internet feitas a partir de: Cáceres, MT, BR, 03 jul. 2013 à 03 fev. 2014].

\begin{tabular}{|c|c|c|c|c|c|c|c|c|c|c|}
\hline \multirow[b]{2}{*}{$\begin{array}{l}\text { CATEGORIAS DE } \\
\text { OBJETIVOS } \\
\text { (No estudo de plantas à } \\
\text { síndrome diabética) }\end{array}$} & \multicolumn{9}{|c|}{ ESPÉCIES } & \multirow[b]{2}{*}{ TOTAL } \\
\hline & 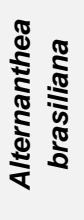 & 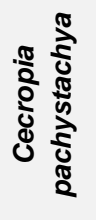 & 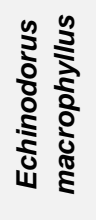 & 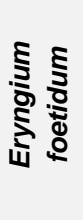 & 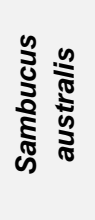 & 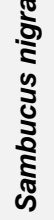 & 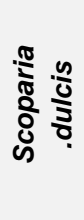 & 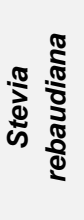 & 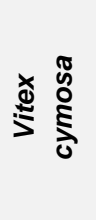 & \\
\hline Verificar efeitos & 2 & 7 & 2 & 1 & - & 2 & 26 & 93 & - & 133 \\
\hline Antidiabético (DM) & - & - & - & - & - & - & 4 & 9 & - & 13 \\
\hline Diurético & - & - & 1 & - & - & 2 & 1 & - & - & 4 \\
\hline Oxidativo & 1 & 1 & - & 1 & - & - & 4 & 8 & - & 15 \\
\hline Glicemiante & - & 1 & - & - & - & - & 2 & 16 & - & 19 \\
\hline Insulínico & - & - & - & - & - & - & - & 3 & - & 3 \\
\hline
\end{tabular}




\begin{tabular}{c|c|c|c|c|c|c|c|c|c|c}
\hline Em feridas & 3 & - & - & - & - & - & - & 2 & - & 5 \\
\hline Sobre peso & - & - & - & - & - & - & - & 7 & - & 7 \\
\hline Toxicidade & - & - & - & - & - & - & 1 & 4 & - & 5 \\
\hline De eficácia e segurança & - & - & - & - & - & - & - & 5 & - & 5 \\
\hline $\begin{array}{c}\text { Outros associados aos } \\
\text { especificados }\end{array}$ & - & 4 & 1 & - & - & - & 4 & 15 & - & 24 \\
\hline $\begin{array}{c}\text { Distintos dos especificados } \\
\text { Etnoconhecimento }\end{array}$ & - & 1 & - & - & - & - & 10 & 24 & - & 35 \\
\hline Etnomedicina & - & 1 & - & 3 & - & - & 11 & 3 & - & 18 \\
\hline Etnobotânico & - & 1 & - & - & - & - & 2 & 2 & 1 & 6 \\
\hline Fitoquímicos & - & - & - & 2 & - & 1 & 1 & 5 & - & 9 \\
\hline $\begin{array}{c}\text { Aplicação em diabetes } \\
\text { (DM) }\end{array}$ & - & 2 & - & - & - & - & 8 & 46 & - & 56 \\
\hline Não identificado & - & - & - & 2 & - & - & 3 & 27 & - & 32 \\
\hline \begin{tabular}{c} 
TOTAL \\
\hline
\end{tabular} & 4 & 13 & 2 & 11 & 1 & 3 & 64 & 177 & 6 & 281 \\
\hline
\end{tabular}

Os textos com propósitos "não identificados" (32=15,38\%) não expressaram objetivos nos seus estudos, porém tiveram aproveitamento devido a citações de aplicação da planta para diabetes. Considerando textos com propósitos identificados $(176=84,61 \%)$ "aplicação da planta para diabetes" predominou $(31,8 \%)$ seguido de "caracterizar o etnoconhecimento" (14,2\%), [etnomedicinal (10,2\%) e etnobotânico (3,4\%)]; Os efeitos visados menos propostos foram o "insulínico" e o de "toxicidade", ambos com 1,7\%.

Trabalhos com objetivo de estudar etnoconhecimento (25) não contemplaram as espécies $A$. brasiliana, $E$. macrophyllus e $S$. nigra, mas foram mais frequentes nas espécies $S$. dulcis $(13=52,0 \%)$ e $S$. rebaudiana $(5=20,0 \%)$.

As proporções de estudos para $S$. dulcis e $S$. rebaudiana e demais espécies se diferenciam nas 15 categorias de objetivos (TABELA 1) encontrados nos estudos publicados ( $\left.\chi^{2} \mathrm{c}=104,75 ; \mathrm{GL}=28 ; \alpha=8,47 \mathrm{E}-11\right)$. As diferenças são mais acentuadas entre a frequência observada (fo) e esperada (fe), nas categorias "abordar etnoconhecimento" em todas as categorias de espécies e; nas categorias objetivadas "avaliar efeito diurético" e "avaliar efeito em feridas", apenas em "demais espécies".

Agrupando em sete, essas 15 categorias, por similaridade, em: a) elaborar etnoconhecimento, b) é ativa na síndrome DM? c) tem outras atividades? d) tóxica? e) apresenta estudo para diabetes, f) revelar composição fitoquímica e, g) objetivo não identificado, foi constatado que as proporções também se diferenciaram quando se cruzaram estas categorias de objetivos com as três de espécies $\left(\chi^{2} \mathrm{c}=47,31 ; \mathrm{G}=12 ; \alpha=4,10 \mathrm{E}-06\right)$. As diferenças mais expressivas entre a frequência observada (Fo) e frequência esperada ( $F e$ ) foram na categoria "etnoconhecimento" para S. dulcis, S. rebaudiana e "demais espécies".

Os 208 textos aproveitados constituíram 281 objetivos. Em média 1,35 por estudo, alguns, portanto, com mais de um em cada estudo. Os objetivos estão desdobrados por espécies conforme segue: 
A.brasiliana: Avaliar perfil ou atividade antioxidante (1), em feridas, cicatrização (1), eficácia de cura (1), práticas para cura (1);

C. pachystachya: Avaliar perfil ou atividade antioxidante (1), hipoglicemiante (1);

E. foetidum: Avaliar o perfil ou atividade antioxidante (1);

S. dulcis: Elucidar o mecanismo molecular de ação hipoglicemiante da planta (1), avaliar efeito hipoglicemiante (2), perfil ou atividade antioxidante (3), sobre estresse oxidativo (1), antidiabético (4), e citotóxico (1);

S. rebaudiana: Avaliar o efeito (atividade) hipoglicemiante (3), no metabolismo da glicose (1), determinar o index glicêmico (1), eficácia comparativa sobre a concentração de glicose (1), de glicosídeos sobre a glicemia (2), avaliar efeito de glicosídeos da planta sobre a diminuição da pressão arterial (1), glicêmico (3), antihiperglicemiante (2), glicêmico em tratamento crônico (1), glicêmico do esteviosídeo em jejum (1), em cobaias submetidas à aloxano sobrecarregadas de glicose e de adrenalina (1), avaliar perfil ou atividade antioxidante (6), sobre estresse oxidativo (1) sobre enzimas antioxidantes hepáticas (1), avaliar efeito ou atividade curativa de enfermidades periodontais (1), eficácia sobre o peso corporal (4), antropométrica de produtos da planta (1), adversos sobre o peso corporal (1), funcional sobre a prevenção e tratamento de sobrepeso (1), antidiabética (3), do consumo da planta sobre diabéticos tipo 2 (1), do uso terapêutico da planta sobre diabéticos tipo (1), extrato da planta sobre diabetes (1), de mistura de plantas sobre diabetes (1), de polifenóis sobre diabetes (1), de hábitos alimentares sobre o desenvolvimento de diabetes tipo 2 (1), citotóxica (3), toxicidade crônica da planta (1), sobre a secreção e metabolismo da insulina (3).

Entre os estudos de etnoconhecimento (25) havia proposições de descrever a relação entre etnobotânica e/ou abordagem etnomedicinal, [mais para S. dulcis e S. rebaudiana].

Juntamente com os objetivos já mencionados estavam incluídos, em alguns estudos, outros enfoques variados dos quais, para $C$. pachystachya (4): validar ou não os usos populares (1); avaliar efeito antiinflamatório (1), antinociceptivo (1), citotóxico (1); para E. macrophyllus (1): investigar efeito protetivo a danos renais induzidos (1); para $S$. dulcis (4): avaliar atividade antibacteriana (1), hepatotoxidez (1); efeitos renais e propriedades diuréticas (1); realizar estudos farmacognóstico da planta (1); para $S$. rebaudiana (15): avaliar efeito ou atividade sobre a síndrome metabólica do diabetes (1), de glicosídeos da planta sobre a pressão arterial (1), sobre as células beta do pâncreas (1), antihiperlipidêmica (1), farmacológica (1), sobre as enzimas hepáticas do metabolismo dos carboidratos (1), sobre a função hepática (2); investigar evidências terapêuticas da planta para diabetes (1); resumir conhecimento atual sobre farmacocinética (1); avaliar perfil lipídico (1), função histológica de vísceras (1); destacar relevância de ingredientes bioativos naturais (1); desenvolver método de extração de alto rendimento de substâncias bioativas (1) e identificar substâncias bioativas (1).

Há estudos com objetivos ainda diferentes dos já especificados. Para C. pachystachya (1): reunir informações relevantes de pesquisas sobre plantas antidiabéticas (1); para $S$. dulcis (10): avaliar efeito antinoceptivo (1), seus potenciais antimicrobianos (1), eficácia da planta com base nos atributos de usos populares (1), os metabólitos secundários em algumas espécies de plantas a fim de contribuir para a sua quimiotaxonomia (1); revisar potencial benéfico desta e de outras espécies (1); identificar substâncias bioativas (1), características químicas e físicas de substâncias bioativas (1); conscientizar a população 
sobre plantas medicinais (1); proteger plantas medicinais (1); propagar o cultivo de plantas medicinais (1); para S. rebaudiana (24): comparar equivalência adoçante de produtos da stevia com outros adoçantes (1), aceitabilidade de alimentos preparados com produtos da stevia com outros adoçantes (1); comparar o efeito da planta e de seus componentes sobre a gliconeogênese (1), produtos medicamentosos (1); avaliar aceitabilidade de fitoterápicos (1), método de extração alto rendimento de substâncias bioativas (1), os rendimentos de técnicas de extração (1), eficácia comparativa sobre a concentração de glicose no sangue (1), efeito sobre doenças cardiovasculares induzidas por diabetes (1), a excreção renal da substância química (1), efeito hepatoprotetor do extrato da planta (1), potencial nutricional (1), perfil lipídico (1), efeito sobre os parâmetros metabólicos (1), presença de diferentes genótipos (1), eficácia comparativa sobre a concentração de glicose no sangue (1); reunir informações relevantes de pesquisas sobre plantas antidiabéticas (1); desenvolver procedimento de extração e filtragem do produto da planta (1), método de extração alto rendimento de substâncias bioativas (1); identificar perfil enzimático presente no soro do fígado (1), tratamento fitoterápico de enfermidades com plantas medicinais (1); investigar a presença de contaminantes químicos em produtos fitoterápicos (1), a participação da planta na excreção renal de glicose (1); comparar efeito do consumo moderado de frutose e de açúcares não calóricos em bebidas sobre o hábito alimentar (1).

A TABELA 2 apresenta, para as nove espécies, uma síntese das conclusões abordadas nos textos considerados, agrupando-as por foco.

TABELA 2. Conclusões abordadas nos textos aproveitados que se referem à fitoterapia no diabetes, com nove espécies utilizadas em Mato Grosso (MT), Brasil (BR), [Buscas na internet feitas a partir de: Cáceres, MT, BR, 03 jul. 2013 à 03 fev. 2014].

\begin{tabular}{|c|c|c|c|c|c|c|c|c|c|c|}
\hline \multirow[b]{2}{*}{$\begin{array}{c}\text { CATEGORIAS DE } \\
\text { CONCLUÕES } \\
\text { ABORDADAS OU } \\
\text { CONSTATADAS SOBRE: }\end{array}$} & \multicolumn{9}{|c|}{ ESPÉCIES } & \multirow[b]{2}{*}{ TOTAL } \\
\hline & 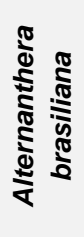 & 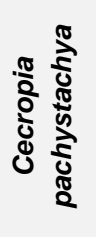 & 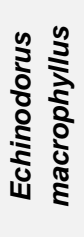 & 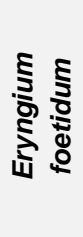 & 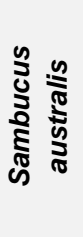 & 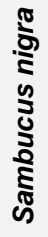 & 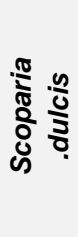 & 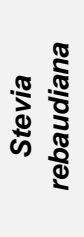 & × & \\
\hline Diabetes & - & - & - & 2 & 1 & 2 & 7 & 3 & 1 & 16 \\
\hline Lipidemiantes & - & - & - & - & - & - & - & 5 & - & 5 \\
\hline Oxidativos & 1 & 1 & - & 1 & - & 1 & 2 & 5 & - & 11 \\
\hline Glicemiantes & - & 2 & - & 1 & - & - & 4 & 23 & - & 30 \\
\hline Insulínicos & - & - & - & - & - & 2 & - & 7 & - & 9 \\
\hline Peso & - & - & - & - & - & - & - & 7 & - & 7 \\
\hline Nutricional & - & - & - & - & - & - & - & 20 & - & 20 \\
\hline Eficácia e segurança & - & - & - & 1 & - & 1 & 1 & 10 & - & 13 \\
\hline $\begin{array}{c}\text { Necessidade de mais } \\
\text { estudos }\end{array}$ & - & - & - & - & - & - & 6 & 6 & - & 12 \\
\hline Feridas & 3 & - & - & - & - & - & - & 1 & - & 4 \\
\hline Etnoconhecimento & - & - & - & - & - & - & 3 & 2 & - & 5 \\
\hline Fitoquímico & - & 1 & - & 2 & - & 1 & 4 & 11 & - & 19 \\
\hline
\end{tabular}




\begin{tabular}{c|c|c|c|c|c|c|c|c|c|c}
\hline $\begin{array}{c}\text { Outros focos associados } \\
\text { aos especificados }\end{array}$ & 2 & 2 & - & - & - & 2 & 13 & 24 & - & 43 \\
\hline $\begin{array}{c}\text { Diferentes dos já } \\
\text { especificados }\end{array}$ & - & 6 & 1 & - & - & - & 7 & 18 & - & 32 \\
\hline $\begin{array}{c}\text { Geografia do uso da planta } \\
\text { Citações de uso } \\
\text { relacionado à DM }\end{array}$ & - & - & - & - & - & - & 4 & - & - & 4 \\
\hline Não identificadas & 1 & 2 & - & - & - & - & 7 & 16 & - & 26 \\
\hline TOTAL & 7 & 15 & 1 & 9 & 1 & 9 & 67 & 204 & 1 & 314 \\
\hline
\end{tabular}

Em 26 textos não foi possível identificar conclusões dos seus respectivos estudos, porém tiveram aproveitamento de algo constante no texto, relacionando a planta ao diabetes. Esta impossibilidade de recuperar as conclusões deve-se, também, a restrições de acesso livre aos sites que disponibilizam os artigos, gerando a categoria "não identificada".

As conclusões "identificadas" (182 estudos) que mais apareceram foram de "citações de uso relacionado à DM" (31,8\%), a exemplo dos objetivos, seguido de efeito "glicemiante" $(16,4 \%)$ e "nutricional" (10,9\%); 26 (14,2\%) estudos não mostraram as conclusões ou não permitiram acesso livre a estes. As conclusões que menos apareceram foram relacionadas a "ação em feridas" e a "geografia do uso da planta" (ambos com 2,19\%).

As proporções da quantidade de estudos para S. dulcis, S. rebaudiana e "demais espécies" se diferenciaram significativamente $\left(\chi^{2} c=93,87 ; G L=32 ; \alpha=5,40 \mathrm{E}-08\right)$ nas 17 categorias de conclusões encontradas nas publicações (TABELA 2). As diferenças foram mais acentuadas entre a frequência observada (fo) e a esperada (fe), na categoria que corresponde a abordagens relacionadas à "feridas" nas "demais espécies" e na categoria "geografia do uso da planta" correspondente à S. dulcis. Agrupando-se essas 17 categorias em outras 10, por similaridade: a) referente ao diabetes, b) efeito glicêmico, c) efeito lipidêmico, d) referentes ao mecanismo da síndrome diabética, e) efeitos em complicações associadas ao dB, f) nutricional e afins, g) outras (atividades, abordagens não farmacológicas, recomendações de mais estudos), h) eficácia e segurança, i) fitoquímica e j) não identificado constatou-se que, as proporções também se diferenciaram significativamente nestas $\left(\chi^{2} \mathrm{c}=50,17 ; \mathrm{GL}=18 ; \mathrm{\alpha}=7,0987 \mathrm{E}-05\right)$. As diferenças mais significantes entre a frequência observada (fo) e frequência esperada (fe) foi na categoria que corresponde a "efeitos em complicações associadas ao diabetes" correspondente à "demais espécies".

De um total de 208 textos aproveitados, houve 314 conclusões, em média 1,50 conclusões por estudo, mais que nos objetivos $(1,35)$.

As conclusões estão descritas, a seguir, para cada espécie estudada.

A. brasiliana: Aumentou a atividade antioxidante (1); melhorou a cicatrização de feridas imunocomprometidas (1), e o efeito na cicatrização de feridas (2);

C. pachystachya: Houve efeito hipoglicemiante (2), antioxidante (1);

E. foetidum: Houve efeito hipoglicemiante (1), antioxidante (1); uso popular para diabetes (2), necessidade de mais estudos para elucidar efeitos antidiabéticos (1); 
S. australis: Constatou-se uso popular para diabetes (1);

S. nigra: Foi verificado potencialização de insulina (1), uso popular para diabetes (2) efeito antioxidante (1), presença de insulina liberada (1), que as flores medicinais poderiam ser incorporadas em bebidas funcionais ou produtos com propriedades bioativas (1);

S. dulcis: Houve efeito hipoglicemiante (2), pode ser útil no tratamento alternativo e complementar da hiperglicemia (1), imita efeitos de dieta sobre hiperglicemia (1), há uso popular para diabetes (4); tem potencial promissor (1), efeito antidiabético (2), antioxidante (1), antiperoxidativo (1);

S. rebaudiana: Houve efeito hipoglicemiante (16); pode ser útil para a comunidade no tratamento alternativo e complementar da hiperglicemia (2), proporcionou efeito glicêmico seguro (1), reduziu o nível de glicose pósprandial (1), melhorou a atividade de tolerância à glicose (1), potencialização (2) e utilização de insulina (2), sendo insulinotrópico (4); raiz da planta é fonte de um tipo de insulina (FOS) (1), planta mostrou potencial promissor (1), ser útil para tratamento de doença periodontal (1), tem efeito antidiabético (1), antioxidante (2); reduziu o peso corporal (5); não houve risco de ganho de peso (2); reduziu o aumento do estresse oxidativo (1); efeito favorável sobre o estresse oxidativo (1), o rebaudiosídeo alivia o estresse oxidativo (1), parece possuir potencial efetivo no tratamento da dislipidemia (3), melhorou o perfil lipídico (1), promoveu alterações desfavoráveis no perfil lipídico devido ao consumo moderado de líquidos com frutose (1).

A planta que mais apresentou conclusões relacionadas a efeitos contra diabetes foi a $S$. dulcis. A planta da qual mais se aproveitou "apenas citações", é a $S$. rebaudiana, e também apresentou 23 conclusões sobre "efeito glicêmico" e 20 "nutricionais". Para V. cymosa: constatou-se uso popular dessa para diabetes.

TABELA 3. Bioatividades encontradas nos textos aproveitados que se referem à fitoterapia no diabetes, com nove espécies utilizadas em Mato Grosso (MT), Brasil (BR), [Buscas na internet feitas a partir de: Cáceres, MT, BR, 03 jul. 2013 à 03 fev. 2014].

\begin{tabular}{|c|c|c|c|c|c|c|c|c|c|c|}
\hline \multirow[b]{2}{*}{$\begin{array}{l}\text { BIOATIVIDADE } \\
\text { DETECTADA }\end{array}$} & \multicolumn{9}{|c|}{ ESPÉCIES } & \multirow[b]{2}{*}{ TOTAL } \\
\hline & 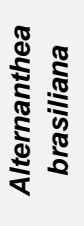 & 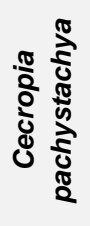 & 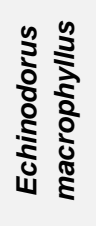 & 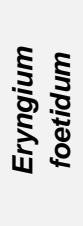 & 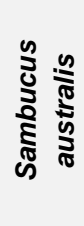 & 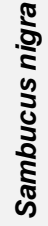 & 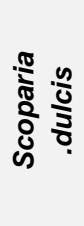 & 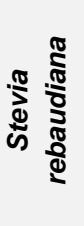 & 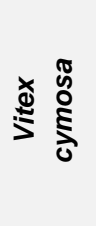 & \\
\hline Favorável & 2 & 3 & 1 & 3 & - & 4 & 26 & 67 & 1 & 108 \\
\hline Não cabe & - & 1 & - & 2 & - & - & 9 & 58 & - & 70 \\
\hline Não favorável & - & - & - & - & - & - & - & 3 & - & 3 \\
\hline Não informado & 1 & 3 & - & 3 & 1 & - & 7 & 13 & - & 27 \\
\hline TOTAL & 3 & 7 & 1 & 8 & 1 & 4 & 42 & 141 & 1 & 208 \\
\hline
\end{tabular}

O estudo sobre a planta $E$. macrophyllus mostrou eficácia na prevenção de lesões renais precoces na diabetes mellitus (PORTELLA et al., 2012), o que se constitui num efeito favorável à saúde. 
De um total de 208 estudos aproveitados, 27 (14,9\%) não informaram a bioatividade detectada; 3 (1,65\%) foram não favoráveis; para 70 (38, 6\%) não cabe essa informação e 108 (59, 6\%) estudos revelaram a bioatividade favorável com relação ao controle do diabetes e complicações. Para as duas espécies $S$. dulcis e $S$. rebaudiana houve predominância de estudos com bioatividade favorável $(26=14,3 \%$ para a primeira e, $67=37 \%$ para a segunda).

$\mathrm{Na}$ espécie S. rebaudiana há estudos que revelam sua inércia sobre a glicose (DYRSKOG, et al., 2005) e também inércia de seus glicosídeos de esteviol (BARRIOCANAL, 2008). Há também recomendação de estudos complementares (SAVITA et al., 2004).

Dos estudos das nove espécies, quatro apresentaram conclusões sobre sua eficácia e/ou segurança para diabetes, sendo elas: E. foetidum (1 não informado), S. rebaudiana (6 favoráveis, 1 não cabe e 3 não informados), S. dulcis (1 não informado) e S. nigra (1 favorável). Segundo Corrêa e Alves (2008), em relação aos medicamentos fitoterápicos, não se pode dizer que o grande diferencial é o custo e, que a vantagem do mesmo está basicamente na ausência de efeitos adversos, ao contrário dos sintéticos.

A prática do uso de plantas medicinais deve ser fortalecida por estudos que comprovem a sua eficácia em seres humanos, contribuindo dessa forma, para estabelecer a toxidez e uso em longo prazo em pacientes diabéticos. Isso pode encorajar o uso tradicional das plantas medicinais ou fazer com que haja uma diminuição a exposição da população a práticas que sejam pouco seguras (SANTOS, NUNES e MARTINS, 2012).

Conforme a TABELA 2, a espécie S. rebaudiana apresenta 23 conclusões de efeito glicemiante, sendo que a mesma sozinha representa $76,6 \%$ destas conclusões. Para as espécies S. dulcis (50\%) e S. rebaudiana (50\%) há necessidade de mais estudos. A espécie S. dulcis apresenta estudos relacionado à geografia do uso da planta, que está associado à sua ocorrência geográfica pelo mundo (NAWAZ et al., 2009), (GUASCH et al., 2012), (OKHALE et al., 2010), (CHAKRAVORTY e GHOSH, 2013).

Em 26 textos não foi possível identificar as conclusões dos seus respectivos estudos, porém tiveram aproveitamento, pois apresentaram citações de uso da planta relacionado à diabetes.

Juntamente com as conclusões já mencionadas, estavam incluídos, em alguns estudos, outros enfoques variados. Assim a $A$. brasiliana (2): incrementou a angiogênese (1), influenciou a fibroplasia (1); $C$. pachystachya (2): diminuiu a atividade mutagênica (1), inibiu a atividade da arginase (1); enquanto $S$. rebaudiana (24): diminui a expressão do gene PEPCK no fígado por ação de abrandamento da gliconeogênese induzida por esteviosídeo (1); apresentou expressão gênica (1); mostrou a distribuição geográfica do uso da planta (1); verificou que a planta usada em diversas partes do mundo como substituto não calórico (1); parece possuir um potencial efetivo no tratamento da hipertensão (2); houve efeito antihipertensivo (1); não alterou a pressão sanguínea (2), os resultados sugerem possibilidade de uso combinado de extrato aquoso de folhas de Stevia e de sementes de Methi (1); houve menor risco de hipoglicemia (1); podem oferecer vantagens terapêuticas (1), restaurou a estrutura de ilhotas de células- $\beta$ (1); a cultura de tecido da stevia melhorou seu rendimento em esteviosídeo propiciando mais oferta para o tratamento da diabetes (1); ausentou propriedades antibacterianas (1); atenuou efeito hepatotóxico (1); não houve efeito colateral (1); a planta é usada em diversas partes do mundo como substituto não calórico (1); participa do mecanismo de ação no transporte da glicose no músculo esquelético (1); baixas concentrações de esteviosídeo indicaram uma iminente ação no sistema de transporte da glicose no músculo esquelético 
(1); produziu ou pode ter efeitos adversos (1); o cultivo da planta em condições locais Ihe conferiram características também específicas (1); o perfil lipídico no plasma como marcador de saúde do fígado (1); ampliou a validade de produtos lácteos e adapta-se bem a condições normais de armazenamento (1); enquanto $S$. dulcis (13): diminui a dor tanto via sistema central como periférico (1); o extrato da planta apresentou moderado efeito bactericida (1) e moderado efeito antifúngico e também um agente anticâncer (1); é um antibiótico mais seguro (1); o extrato da planta apresentou significante atividade citotóxica (1); outras plantas podem ter substâncias novas permitindo o desenvolvimento de novas drogas mais eficazes e com menos efeitos adversos (1); pode ser um inibidor potente da enzima a-glicosidase humana e assim retardar a digestão de amido e de sacarose (1); reduziu a glicemia pós prandial (1); o extrato metanólico e hexânico inibiu o crescimento de fungos (1) e o crescimento de três bactérias (1); o extrato da planta é ativo contra alguns microorganismos (1); práticas medicinais populares são comuns entre áreas fronteiriças de Bangladesh e Índia (1); a comunidade tem conhecimento das plantas de cerrado para curar doenças (1). Por sua vez a $S$. nigra (2): é importante e útil em países cujo povo tem acesso limitado à medicamentos modernos (2)

Há ainda estudos com conclusões diferentes das já especificadas. Para E. macrophyllus (1): possui efeito nefroprotetor (1); para C. pachystachya (6): mostra a distribuição geográfica do uso da planta (1), métodos de extração mais usados como o solvente (1), técnicas de extração de engenharia, como a extração supercrítica e microondas assistida (1); folhas são as partes mais usadas (1), fontes de estudos sobre plantas antidiabéticas, com valor terapêutico identificados, indicam que a Ásia tem predominância, seguido da África (1); inibiu as células tumorais (1); para $S$. rebaudiana (18): são métodos de extração mais eficazes (1); revitalizou células- $\beta$, antagonizando a ação necrótica do aloxano (1); verificou que as folhas da planta podem promover equilíbrio calórico beneficiando um estilo de vida alimentar saudável (1); ficou contestada ausência de toxidez (1); os muitos anos de uso não causaram efeitos secundários significativos (1); os esteviosídeos e análogos são baratos para produzi-los para tratamento da diabetes e síndrome metabólica (1); melhorou o conhecimento sobre a conservação da planta (1) e sobre a planta (1); o material utilizado manteve-se estável organolepticamente em preparados para chá (1); verificou que a cooperação entre pessoas e empresas facilita, melhora e aumenta o desenvolvimento destas e de sua produção (1); desenvolveu-se um método de extração MAE (1); o extrato aquoso da planta não afetou a reprodução (1); o extrato metanólico é fonte de antioxidantes (1); plantas medicinais devem ser coletadas em ambientes não poluídos (1); os materiais vegetais tem atividade sequestradora de radicais livres (1), há secreção de steviol na urina e esclarece o mecanismo de secreção (1); há sugestão sobre formas de atuar no mercado de plantas medicinais para otimizar toda a cadeia do processo produtivo (1); propõem modelagem ANN que podem ser usadas para se obter, com boa precisão, uma previsão de substâncias bioativas, para os processos de extração de faixa de trabalho amplo (1); para $S$. dulcis (7): recomendaram-se estudos fitoquímicos para descoberta de novas drogas (1); supõe que os novos princípios ativos isolados da planta podem resultar em um novo e eficaz tratamento padrão (1); tem atividade pró coagulação e este foi mais rápida do que a vitamina $\mathrm{K}$ (1); ervas daninhas podem ter valor medicinal (1); os estudos contribuem para conhecer melhor as plantas medicinais, seu etnoconhecimento e usos, assim como para estimular e orientar a conservação de espécies (1); houve atividade hepatoprotetora (1); potencial de eliminação de radicais livres (1). 


\section{Considerações finais}

Os 208 textos aproveitados continham 281 objetivos (1,35 textos/objetivo). A categoria "aplicação da planta para diabetes" foi mais ocorrente $(31,8 \%)$, e "caracterizar o etnoconhecimento" [etnomedicinal e etnobotânico] teve apenas $14,2 \%$ dos casos.

Alguns estudos (32) os objetivos não foram explícitos, porém foram aproveitados por terem citações de aplicação da planta para diabetes.

Os objetivos menos visados foram as categorias "verificar efeito insulínico" e o de "verificar a toxicidade".

As 15 categorias de objetivos nas investigações com a $S$. dulcis, S. rebaudiana e "demais espécies" se diferenciaram significativamente em suas proporções de ocorrência. As diferenças mais acentuadas ocorreram na categoria de "etnoconhecimento". No grupo "demais espécies" as diferenças mais acentuadas ocorreram nas categorias "avaliar efeito diurético" e "avaliar efeito em feridas". Reagrupando por similaridade, essas 15 categorias em 7, as novas proporções também se diferenciaram nos cruzamentos de objetivos com as três de espécies. As diferenças mais expressivas foram na categoria "etnoconhecimento" tanto para S. dulcis e S. rebaudiana como para as "demais espécies".

Para 208 textos aproveitados, houve 314 conclusões (1,50 texto/conclusão). A categoria "citações de uso relacionado à diabetes" foi a mais ocorrente (31,8\%), seguido de "efeito glicemiante" $(16,4 \%)$. Em 26 textos não foi possível identificar conclusões dos seus respectivos estudos, porém tiveram aproveitamento (incluídos na análise geral) para algum aspecto aqui visado, tal como "objetivos".

As proporções da quantidade de estudos para S. dulcis, S. rebaudiana e "demais espécies" se diferenciaram significativamente. As diferenças foram mais acentuadas na categoria que corresponde a abordagens relacionadas à "feridas" nas "demais espécies" e, na categoria "geografia do uso da planta" correspondente à S. dulcis. Reagrupando essas 17 categorias, por similaridade, as proporções também se diferenciaram significativamente nestas. As diferenças mais significantes foram na categoria que corresponde a "efeitos em complicações associadas ao diabetes" nas "demais espécies".

Dos 208 estudos, 111 mostraram bioatividade e 108 foram favoráveis à saúde. Em três estudos, há revelações de que a $S$. rebaudiana apresenta inércia farmacológica sobre a glicose (1), inércia de seus glicosídeos de esteviol (1) e há recomendação de estudos complementares (1).

As espécies $S$. austalis e $V$. cymosa revelaram "uso popular para a diabetes"; para $S$. nigra verificou a potencialização da insulina; para $A$. brasiliana, houve efeito na cicatrização de feridas e para as espécies: C. pachystachya, E. foetidum, S. dulcis e S. rebaudiana houve efeito hipoglicemiante. Já a E. macrophyllus mostrou prevenir lesões renais precoces em diabéticos. A planta S. rebaudiana também mostrou ser promissora na redução de glicose pós-prandial e efeito antidiabético. A mesma ainda demonstrou redução do peso corporal e que não há risco de ganho de peso.

Os maiores números de estudos estão centrados em $S$. dulcis e $S$. rebaudiana, e com predominância também de objetivos e de conclusões. Já para as demais, o número de estudos é relativamente baixo, o que acaba estimulando novas pesquisas e aprofundamento de conhecimentos, propiciando novas descobertas. 


\section{Referências}

BARRIOCANAL, L. A.; MAFALDA, P.; BENITEZ, G.; BENITEZ, S.; JIMENEZ, J. T.; JIMENEZ, N.; ROJAS. $\mathrm{V}$. Apparent lack of pharmacological effect of steviol glycosides used as sweeteners in humans. A pilot study of repeated exposures in some normotensive and hypotensive individuals and in Type 1 and Type 2 diabetics. Elsevier. Regulatory toxicology and pharmacology, v. 51, n. 1, p. 37-41, USA. 2008. ISSN 02732300. [CrossRef] [PubMed].

CHAKRAVORTY, A.; GHOSH, P. D. Exploration of the otherwise-neglected weeds of Nadia district-the treasure trove of medicines. International Journal of Pharmaceutical Science Invention, v. 2, n. 6, p. 19-21, USA. 2013. ISSN 2319-6718. [Link].

CHAVES, D. S. A. As plantas medicinais brasileiras são eficazes no tratamento do lúpus eritematoso sistêmico? Farmanguinhos/Fiocruz. Revista Fitos. v. 7, n. 3, p. 198-206, Rio de Janeiro. 2012. [Link].

COMPLEMENTARY MEDICINE (CAM) - Its current position and its potential for European healthcare, 2008. Disponível em: [Link]. Acesso em: 23 abr. 2015.

CÔRREA, C. C.; ALVES, A. F. Plantas medicinais como alternativa de negócios: caracterização e importância. 46th Congress, Sociedade Brasileira de Economia, Administração e Sociologia Rural (SOBER), Rio Branco. 2008. [Link].

DYRSKOG, S.E.U.; JEPPESEN, P. B.; COLOMBO, M.; ABUDULA, R.; HERMAN, K. The diterpene glycoside, rebaudioside $A$, does not improve glycemic control or affect blood pressure after eight weeks treatment in the Goto-Kakizaki rat. Society for Biomedical Diabetes Research. The Review of Diabetic Studies, v.2, n. 2, p. 84-91, USA. 2005. ISSN 1613-6071. [CrossRef] [PubMed].

GUASCH, L.; SALA, E.; OJEDA, M. J.; VALLS, C.; BLADÉ, C.; MULERO, M. Identification of novel human dipeptidyl peptidase-IV inhibitors of natural origin (Part II): in silico prediction in antidiabetic extracts. PloS One, v.7, n. 9, p. 1-7, USA. 2012. [CrossRef] [PubMed].

MAZZOLIN, L. P. Avaliação da atividade antioxidante de qualea parviflora sobre a doença inflamatória intestinal e a úlcera gástrica induzidas em roedores. Tese de Doutorado apresentada no Instituto de Biociências, UNESP, Botucatu, 2013.

NAWAZ, A.H.M.M.; HOSSAI, M.; KARIM, M.; KHAN, M.; JAHAN, R.; RAHMATULLAH, M. An ethnobotanical survey of Rajshahi district in Rajshahi division. AENSI Publication. American Eurasian Journal of Sustainable Agriculture, v. 3, n. 2, p. 143-150, Bangladesh. 2009. ISSN 1995-0748. [Link].

NEGRI, G. Diabetes mellitus: plantas e princípios ativos naturais hipoglicemiantes. SciElo. Revista Brasileira de Ciências Farmacêuticas, v. 41, n. 2, p. 121-142, São Paulo. 2005. ISSN 15169332. [CrossRef].

OKHALE, S. E.; AMANABO, M. O.; JEGEDE, I. A.; EGHAREVBA, H. O.; MUAZZAM, I. W.; KUNLE, O. F. Phytochemical and Pharmacognostic Investigation of Antidiabetic Scoparia dulcis Linn Scrophulariaceae Whole Plant Grown in Nigeria. Researcher, v.2, n. 6, p. 7-16, USA. 2010. ISSN: 1553-9865. [Link]. 
ORGANIZAÇÃO MUNDIAL DA SAÚDE. Estratégia de la OMS sobre medicina tradicional. Genebra: OMS, 2002-2005. Disponível em: [Link]. Acesso em: 23 abr. 2015.

PORTELLA, V.G.; COSENZA, G.P.; DINIZ, L.R.L.; PACHECO, L.F.; CASSALI, G.D.; CALIARI, M.V. Nephroprotective Effect of Echinodorus macrophyllus Micheli on Gentamicin-Induced Nephrotoxicity in Rats. Karger. Nephron extra, v. 2, n. 1, p. 177-183, USA. 2012. ISSN 1664-5529. [CrossRef]. [PubMed].

RIEDER, A.; GUARIM NETO, G. Saúde e ambiente: plantas medicinais utilizadas para o controle de diabetes em Mato Grosso, Brasil. Editora UNEMAT. Cáceres/Mato Grosso, 2012. ISBN 978-85-7911-074-0.

RIEDER, A.; RODRIGUES F.A.C. Recuperação e filtragem de textos sobre saúde e ambiente através de mecanismos de busca na internet. Revista Informação e Sociedade, 2012 (aguardando publicação).

SANTOS, M.M.; NUNES, M.G.S.; MARTINS, R.D. Uso empírico de plantas medicinais para tratamento de diabetes. SciElo. Revista Brasileira de Plantas Medicinais, v. 14, n. 2, p.327-334, Paulínia. 2012. [CrossRef] [Link].

SAVITA, S. M.; SHEELA, K.; SUNANDA, S.; SHANKAR, A. G.; RAMAKRISHNA, P.; SAKEY, S. Health implications of Stevia rebaudiana. Journal of Human Ecology, v.15, n. 3, p.191-194, Delhi, India. 2004. ISSN 0970.9274. [Link].

SOCIEDADE BRASILEIRA DE DIABETES. Diretrizes da Sociedade Brasileira de Diabetes-SBD. AC. Farmacêutica, São Paulo, 2016. ISBN 978-85-8114-307-1. Disponível em: [Link]. Acesso em: 17 nov. 2016.

TELESI JUNIOR, E. Práticas integrativas e complementares em saúde, uma nova opção para o SUS. USP. Estudos Avançados, v. 30, n. 86, p. 99-112, São Paulo. 2016. ISSN 1806-9592. [CrossRef].

YUNES, R. A.; PEDROSA, R. C; CECHINEL FILHO, V. Fármacos e fitoterápicos: a necessidade do desenvolvimento da indústria de fitoterápicos e fitofármacos no Brasil. SBQ. Química nova, v. 24, n. 1, p. 147-152, São Paulo. 2001. [CrossRef] [Link].

Conflito de interesses: O presente artigo não apresenta conflitos de interesse.

Histórico do artigo: Submissão: 29/02/2016 | Aceite: 25/01/2017 | Publicação: 23/05/2017

Como citar este artigo: SANTOS, Ana Paula A. dos; RIEDER, Arno. Análise de objetivos e conclusões de estudos com nove plantas usadas para o controle de diabetes em Mato Grosso. Revista Fitos. v.10, n.4. p. 383-396. Rio de Janeiro. 2016. e-ISSN 2446.4775. Disponível em: <http://revistafitos.far.fiocruz.br/index.php/revista-fitos/article/view/311>. Acesso em: 09 maio 2017.

Licença CC BY 4.0: Você está livre para copiar e redistribuir o material em qualquer meio; adaptar, transformar e construir sobre este material para qualquer finalidade, mesmo comercialmente, desde que respeitado o seguinte termo: dar crédito apropriado e indicar se alterações foram feitas. Você não pode atribuir termos legais ou medidas tecnológicas que restrinjam outros autores de realizar aquilo que esta licença permite. 